\title{
Determination of any pure spatial qudits from a minimum number of measurements by phase stepping interferometry.
}

\author{
Quimey Pears Stefano, ${ }^{1,2, \text { * }}$ Lorena Rebón, ${ }^{3}$ Silvia Ledesma,,${ }^{1,2}$ and Claudio Iemmi ${ }^{1,2}$ \\ ${ }^{1}$ Universidad de Buenos Aires, Facultad de Ciencias Exactas y Naturales, \\ Departamento de Física, Buenos Aires, Argentina \\ ${ }^{2}$ Consejo Nacional de Investigaciones Científicas y Técnicas, Buenos Aires, Argentina. \\ ${ }^{3}$ Departamento de Física, IFLP-CONICET, Universidad Nacional de La Plata, C.C. 67, 1900 La Plata, Argentina.
}

(Dated: December 11, 2017)

\begin{abstract}
We present a proof-of-principle demonstration of a method to characterize any pure spatial qudit of arbitrary dimension $d$, which is based on the classic phase shift interferometry technique. In the proposed scheme a total of only $4 d$ measurement outcomes are needed, implying a significant reduction with respect to the standard schemes for quantum state tomography which require of the order of $d^{2}$. By using this technique, we have experimentally reconstructed a large number of states ranging from $d=2$ up to 14 with mean fidelity values higher than 0.97 . For that purpose the qudits were codified in the discretized transverse momentum-position of single photons, once they are sent through an aperture with $d$ slits. We provide an experimental implementation of the method based on a Mach-Zehnder interferometer, which allows to reduce the number of measurement settings to 4 since the $d$ slits can be measured simultaneously. Furthermore, it can be adapted to consider the reconstruction of the unknown state from the outcome frequencies of $4 d-3$ fixed projectors independently of the encoding or the nature of the quantum system, allowing to implement the reconstruction method in a general experiment.
\end{abstract}

\section{INTRODUCTION}

Determining the state of a quantum system is one of the fundamental tasks in quantum information processing and a recurrent problem in quantum mechanics [1]. In this regard, quantum state tomography provides a mean of fully reconstructing the density matrix which describes the state of a quantum system. For typical quantum state tomography methods [2 [5] the number of required measurements settings (or outcomes) increases with the dimension of the system $d$, as $d^{2}$, that makes difficult the treatment of high-dimensional quantum systems. Therefore, as diverse applications of quantum information can be enhanced using a dimension greater than two [6-10], there is a growing interest in estimating $d$-level quantum systems (qudits) from a reduced number of measurements.

With some a priori information of the unknown quantum system, a reduction in the number of measurements is feasible. For example, in case of pure or nearly pure quantum states, compressed sensing techniques allow obtaining, with high probability, the reconstruction of the state with a number of measurements of the order of $d(\log d)^{2}$ [11, 12]. This technique works by randomly choosing a set of observables and measuring their expectation values. Thus, it does not provide an explicit measurement set-up. Besides, the amount of measurements is still far to be optimal.

Flammia et al. 13 had established that a measurement with at least $2 d$ outcomes is required to determine almost all (but not all) pure states. Furthermore, they

\footnotetext{
* quimeyps@df.uba.ar
}

have also demonstrated that $3 d-2$ one-dimensional projectors are sufficient for determining a generic pure state, with the exception of a set of measure zero. This number increases if we want to distinguish any two pure states. In such a case, a measurement with $\sim 4 d$ outcomes must be considered [14], or when restricting to projective measurements, at least four orthonormal bases are required if $d \geq 3$, except maybe for $d=4$, in which case it is not known whether three bases would be sufficient [15]. However, the measurements do not provide a way of verifying the purity assumption.

Recently, Goyeneche et al. [16] have proposed a method to determine an arbitrary pure state of any dimension by means of projective measurements onto five fixed orthonormal bases, resulting in a total of $5 d$ measurements outcomes. They have experimentally implemented the method for reconstructing spatial qudits [17. The measurement settings required for that scheme could be interpreted as equivalent to a four step phase shifting interferometry (PSI) between pairs of consecutive slits. As it is well known PSI leads to the most accurate way to measure the amplitude and phase distribution of a wavefront 18 . In these techniques controlled phase displacements are introduced between the reference and the object beam, then the wavefront under test can be determined from the interferograms corresponding to the different phase shifts. The number of interferograms to be recorded, as the phase is shifted, varies depending on the algorithm employed to recover the phase distribution of the wavefront. Typically, four or three step algorithms are used.

In dimension $d=2$, the connection between quantum state tomography and PSI was studied by Rebón et al. [19]. They showed that for this particular case the full quantum tomography of any arbitrary qubit, pure 
or mixed, is equivalent to a four step PSI. In that work, a path-qubit was codified as the superposition state of a single-photon occupying two arms of a Michelson interferometer. The PSI was carry out by obtaining the different interferograms between both paths with one of them as the reference.

In this article we propose a quantum state estimation method, based in a three step PSI algorithm, that allows to determine any pure spatial qudit of arbitrary dimension $d$ by means of a minimum number of measurements. In fact, in our method the number of measurement bases is 4 , which is lower than the number of bases requiered in Ref. [16], and even more, it is consistent with the minimum number of measurement outcomes reported in [13 15. In Section II we provide a complete description of the qudit estimation process and point out how the measurements onto these 4 basis are also sufficient for verifying the purity assumption of the unknown state. In addition, for photonic qudits codified in the transverse momentum-position of single photons, we provide an experimental implementation of the method based on an interferometer scheme. Our setup allows to reduce the number of measurement settings to only 4, regardless of the dimension $d$ of the system. The results are presented and discussed in Section III, before going into the conclusions.

\section{METHOD}

The encoding process of the $d$-dimensional quantum system is performed in the discretized transverse momentum of single photons once they are sent through an aperture with $d$ slits [20, 21]. Such pure state can be expressed as

$$
|\Psi\rangle=\sum_{k=0}^{d-1} c_{k}|k\rangle,
$$

where the $c_{k}$ 's are the complex coefficients that represent the complex transmission amplitude of each slit, and $|k\rangle$ denotes the state of the photon passing through the slit $k$. These coefficients can be explicitly written as $c_{k}=\left|c_{k}\right| e^{i \varphi_{k}}$ where $\varphi_{k}$ represents the argument of the complex number $c_{k}$. For reconstructing the quantum state of these systems we use one of the slits as a phase reference and implement the three step PSI algorithm to find the phase of each of the remaining slits with respect to the reference, that is finding the argument $\varphi_{k}$. The additional measurement of the intensity of each slit allows the unambiguous reconstruction of the state up to an arbitrary global phase and also give us a way to certify if the state is pure - or nearly pure- without any a priori assumptions. The total number of measurements outcomes in this method is $4 d-3$ when the procedure is performed in an adaptive way, or $4 d$ in case of fixed measurement settings. Even more, the proposed experimental setup for reconstructing spatial qudits has the

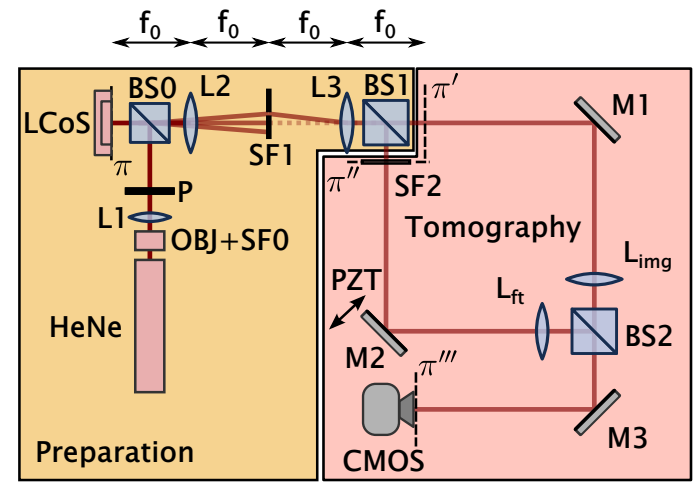

FIG. 1. Experimental setup for reconstructing pure spatial qudits. Preparation part: an expanded and collimated HeNe laser impinges onto a phase only LCoS modulator. In conjunction with the $4 f$ processor formed by the lens $\mathbf{L}_{\mathbf{2}}$ and $\mathbf{L}_{\mathbf{3}}$ and the spatial filter SF1 the quantum state is encoded in the planes $\pi^{\prime}$ and $\pi^{\prime \prime}$. Tomography part: A lens $\mathbf{L}_{\text {img onto }}$ the Image arm of the Mach Zender interferometer images the plane $\pi^{\prime}$ onto the output plane $\pi^{\prime \prime \prime}$. The lens in the Fourier arm $\mathbf{L}_{\mathrm{ft}}$ perform the Fourier Transform of the only slit that is not blocked by the spatial filter SF2 .

advantage that every of the four sets of $d$ measurements corresponds to a single interferogram, thus, using photon counting cameras 22] instead of a point-like single photon detection module (SPDM), $d$ measurements can be recorded in only one acquisition, i.e. only 4 pictures are needed, in any dimension $d$, to determine the unknown state. Nevertheless, the set of $4 d-3$ quantum projectors to be used in order to perform the tomographic process do not depend on the particular encoding or the nature of the quantum system and they could be applied in a completely general setup.

Let us to start by briefly describing the state preparation which is carried on by using the first part of the optical setup sketched in Fig. 1. The light source is an HeNe laser that is expanded, filtered, and collimated by the objective $\mathbf{O B J}$, the spatial filter $\mathbf{S F O}$ and the lens $\mathbf{L}_{\mathbf{1}}$. To test the proposed method at the single-photon level we inserted neutral-density filters to highly attenuate the power of the laser beam to $0.005 \mathrm{nW}$. It implies that, for an interferometer with a total length of $140 \mathrm{~cm}$ as in our case, less than one photon on average is present, at any time, in the experiment. This source can be used to mimic the single-photon qudit state given by Eq. (1), and as is usual in optical implementations of quantum-states estimation, it is enough to test the feasibility of the proposed method 23 25]. The beam that impinges on the spatial light modulator used to codify the slit states, has approximately constant amplitude and phase over the regions of interest (ROIs) where the slits are displayed. The method for codifying arbitrary complex amplitudes of spatial photonic qudits was developed for our group in previous works [26, 27]. We briefly explain here the main features of the method: Blazed phase gratings are displayed onto each slit region. The real amplitude of the 
slit is determined by the diffraction efficiency achieved through the phase modulation of the grating. On the other hand the desired phase value is obtained just by adding an adequate constant phase. The required pure phase modulation is provided by a parallel aligned liquid crystal on silicon display (LCoS) Holoeye PLUTO with HDTV resolution (1920x1080) and pixel size of $8 \mu \mathrm{m}$. In our case the width of the slits is 10 pixels, and the separation between slit centers is 30 pixels. In order to implement the mentioned codification we use a typical $4 f$ processor conformed by lenses $\mathbf{L}_{\mathbf{2}}$ and $\mathbf{L}_{\mathbf{3}}\left(f_{0}=20 \mathrm{~cm}\right)$. The spatial period of the gratings displayed onto the slit regions is 16 pixels which is enough to select by means of the spatial filter SF1 the first diffracted order. This optical setup together with the non polarizing beam splitter BS1 allows to obtain on planes $\pi^{\prime}$ and $\pi^{\prime \prime}$ the desired complex amplitude distribution.

The tomographic process employed to characterize the $d$-dimensional spatial qudit is implemented by using the Mach-Zehnder interferometer schematized in the second part of Fig. 1. Let us call Image Arm (IA) the one that contains lens $\mathbf{L}_{\text {img }}$. This lens in configuration $2 f-2 f$ $\left(f_{\text {img }}=35 \mathrm{~cm}\right)$ images the input state obtained on $\pi^{\prime}$ over the final plane $\pi^{\prime \prime \prime}$. Meanwhile the Fourier Arm (FA) is the one that contains the lens $\mathbf{L}_{\mathrm{ft}}$ in configuration $f-f\left(f_{\mathrm{ft}}=70 \mathrm{~cm}\right)$ giving the exact Fourier Transform of plane $\pi^{\prime \prime}$ over $\pi^{\prime \prime \prime}$. The spatial filter SF2 (a slit of width $200 \mu \mathrm{m}$ ), placed on plane $\pi^{\prime \prime}$, blocks all but one slit that acts as reference. The resulting output is the interference pattern between the complex amplitude of the $d$ slits and the reference. Finally, intensity measurements are carried out by means of a high sensitive camera based on CMOS technology placed in $\pi^{\prime \prime \prime}$. The camera used is an Andor Zyla 4.2 sCMOS.

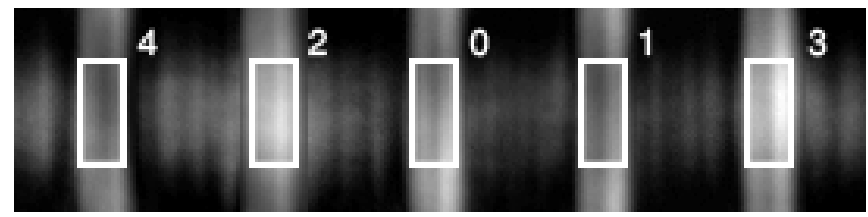

FIG. 2. Interferogram for a state of dimension $d=5$. The vertical lighted bands correspond to the image of the five slits. The horizontal lighted band corresponds to the Fourier Transform of the filtered slit in the FT arm of the interferometer, which acts as the reference. The rectangles indicate the regions on which the measurements are performed, and $0,1, \ldots, 4$ are the corresponding slit numbers.

As an example, one of the interferograms obtained at the output of the Mach-Zender for a qudit of dimension $d=5$ is shown in Fig. 2. The lighted bands in the vertical direction correspond to the image of the five slits. The lighted band in the horizontal direction is the Fourier Transform of the only slit that is not blocked by SF2 in the FA of the interferometer. This is the slit that acts as the reference. The drawn rectangles delimit the ROIs on which the measurements are performed.

It is important to note that a similar implementation of the reconstructing method can be done with exactly the same setup by using a SPDM which must be displaced over the final plane $\pi^{\prime \prime \prime}$ in order to measure sequentially the counts in the different ROIs. However, the use of high sensitive cameras, that increasingly become an interesting option for single photon detection in quantum optics experiments [28 30, makes possible to complete the measurement stage by taking 4 snapshot, no matter the dimensionality of the unknown spatial qudit. This is possible both due to the proposed setup which enables to perform a simultaneous detection of the $d$ regions (see Fig. 22 as well as the selected PSI scheme. In fact, a simultaneous measurement is not possible by using the set of measurement bases presented in [16] since in such a case, the tomographic process is equivalent to a PSI scheme which requires the sequential interference of contiguous slits, i. e., there is not an unique reference beam as in our case.

We will now proceed to analyze the tomographic reconstruction method. In order to characterize the quantum state in Eq. (1) it is necessary to know the complex amplitudes $c_{k}$, i.e., the amplitude and phase of the wavefront just in the region the slit $k$. To this end we implemented the classical PSI technique of three steps, involving successive phase shifts of $\pi / 2$ that were introduced in the reference arm of the interferometer by means of the piezoelectric actuator PZT. The recorded intensities of the interferograms corresponding to the different phase shifts, can be described as [18]:

$$
\begin{aligned}
I_{\ell}(x, y)= & I_{0}(x, y)\{1+ \\
& \left.\gamma(x, y) \cos \left[\varphi(x, y)-\frac{\pi}{4}+\frac{\pi}{2} \ell\right]\right\}, \ell=1,2,3
\end{aligned}
$$

where $(x, y)$ represents the transverse position in the output plane $\pi^{\prime \prime \prime}, I_{0}(x, y)$ is the arithmetic sum of the intensity of the light beams in each arm of the interferometer, $\varphi(x, y)$ is their relative phase and $\gamma(x, y)$ is the modulation of the interference fringes. From these three interferograms it is possible to obtain the relative phase of the object beam (IA) with respect to the reference beam (FA), at every point of $\pi^{\prime \prime \prime}$

$$
\varphi(x, y)=\tan ^{-1}\left(\frac{I_{3}(x, y)-I_{2}(x, y)}{I_{1}(x, y)-I_{2}(x, y)}\right) .
$$

In our case, the phase over each slit region should be a constant. However, there exist slight variations $(\sim 2 \%)$ mainly due to inhomogeneities of the LCoS display used as SLM, so we have taken as argument of the coefficient $c_{k}$ in Eq. (1), the average of the obtained phase, $\varphi_{k}=$ $\overline{\varphi(x, y)}$, over the interference region assigned to the slit $k$ (see Fig. 2). It should be considered that when applying the PSI algorithm the recovered phase is not $\varphi_{k}$ but $\varphi_{k}-$ $\varphi_{0}$. Hence, for reconstructing the quantum state up to a global phase, we can always define the phase of the reference slit, $\varphi_{0}$, as zero. The modulus of the coefficients 
$c_{k}{ }^{\prime} s$ correspond to the square root of the slit intensities and can be obtained just by blocking the reference arm and averaging over the same ROIs.

It is obvious that the slit selected as reference, for a given quantum state, must have a non-null intensity value. It means that the presented algorithm fails when the quantum state to be determined has a null coefficient $c_{0}$. To prevent such a case, a possibility is to first measure the intensity of the $d$ slits and obtain the modulus of each coefficient, $\left|c_{k}\right|$; then, the slit with the greater intensity value can be selected as the reference and accordingly, adjusting the position of SF2. The drawback of this strategy is that the reference must be redefined every time, what entails to change the filter position and realign the setup during the measurements. In order to avoid that, which is experimentally not convenient and timeconsuming, we adopted an alternative possibility which consists in adding an extra slit with maximum transmission amplitude to be used as reference, totalling $d+1$ slits of which only $d$ are used to codify the state. Hence, we are able to reconstruct arbitrary pure states without changing the experimental configuration. Besides, with the addition of these intensities measurements, we can distinguish between pure and mixed states. Pure states are characterized by interference patterns with maximum visibilities (bounded to the ratio of intensities between interfering beams) and denote maximum coherence between any pair of slits, whose value can be easily obtained from the set of measurements outcomes [18].

\section{RESULTS AND DISCUSSION}

To evaluate the viability of the method and the quality of the proposed setup we performed the reconstruction of a large number of pure states, taking as examples systems of dimension $d=2$ and $d=14$. As figure of merit, we calculated the fidelity $F \equiv \operatorname{Tr}(\sqrt{\sqrt{\varrho} \rho \sqrt{\varrho}})$, between the state intended to be prepared, $\varrho$, and the density matrix of the reconstructed state, $\rho$ [31. Ideally, $F=1$. Figure 3 represents the obtained fidelities for 1024 qubits $(d=2)$ uniformly distributed in the surface of the Bloch sphere. The mean value of the fidelity is $\bar{F}=0.997$, and the standard deviation $\sigma_{F}=0.003$. The histogram in Fig. 4 shows the occurrence of the fidelities for 250 states of dimension $d=14$ randomly chosen. The average fidelity is $\bar{F}=0.98$ while the standard deviation is $\sigma_{F}=0.01$. In this high-dimensional case the mean fidelity is only slightly lower than in the bidimensional case. A similar behaviour was observed for qudits of intermediate dimensions not shown here. Then, the limitation of the experimental setup for implementing the reconstruction method is the amount of slits that falls under the central diffraction pattern of the reference slit. In order to verify the purity of the states we have compared the actual visibility, obtained from $\gamma(x, y)$, with the expected value for a pure state that can be calculated from the intensity measurements. We have observed that they overlap

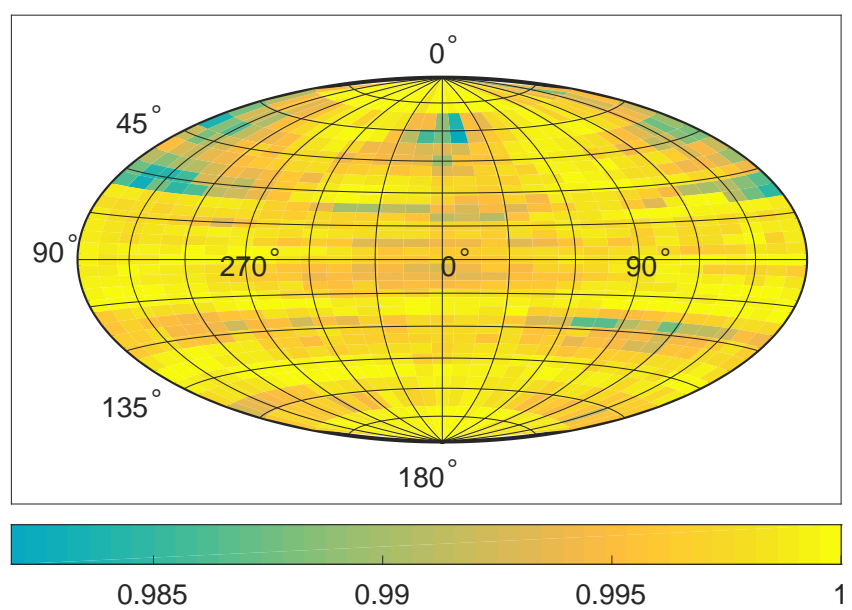

FIG. 3. Bloch sphere showing the reconstruction fidelities of 1024 states uniformly distributed on the surface. The mean value of the fidelity is $\bar{F}=0.997$, and the standard deviation $\sigma_{F}=0.003$.

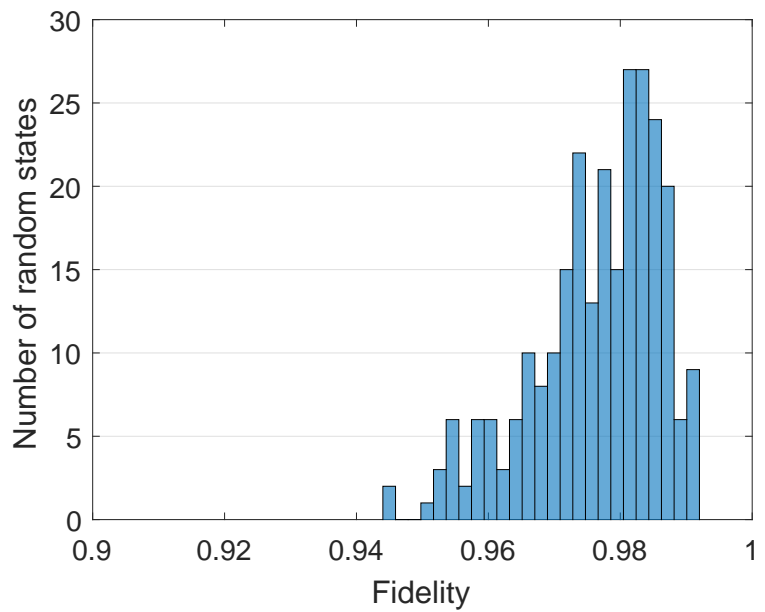

FIG. 4. Histogram of the reconstruction fidelities for 250 random states in $\mathrm{d}=14$. The mean value of the fidelity is $\bar{F}=0.98$, and the standard deviation $\sigma_{F}=0.01$.

within the experimental errors.

It is worth to note the relation between the classical PSI steps and quantum projectors. For every slit $k$ which defines the state $|k\rangle$ of the canonical base - except the reference, we can define a set of three $d$-dimensional states

$$
\left|\Psi_{\ell}^{(k)}\right\rangle=\frac{|0\rangle+e^{i \pi / 2 \times(\ell-1 / 2)}|k\rangle}{\sqrt{2}}, \ell=1,2,3
$$

where $|0\rangle$ represents the reference slit, and $k$ runs from 1 to $d-1$. These states show the same phase relation between the reference and the target slit that the phase shifts introduced in the three step PSI. To each of these states we can associate a projector $\hat{\mathrm{P}}_{\ell}^{(k)}=\left|\Psi_{\ell}^{(k)}\right\rangle\left\langle\Psi_{\ell}^{(k)}\right|$. 
The outcome probabilities of this set of projectors, $p_{\ell}^{(k)}=$ $\left\langle\Psi\left|\hat{\mathrm{P}}_{\ell}^{(k)}\right| \Psi\right\rangle=\left|\left\langle\Psi_{\ell}^{(k)} \mid \Psi\right\rangle\right|^{2}$, are given by the following expression, totally analogue to those described in Eq. (2),

$$
p_{\ell}^{(k)}=\frac{\left|c_{0}\right|^{2}}{2}+\frac{\left|c_{k}\right|^{2}}{2}+\Re\left\{c_{0} c_{k}^{*} e^{i \pi / 2 \times(\ell-1 / 2)}\right\} .
$$

With the knowledge of $c_{0} \equiv+\sqrt{p_{0}}>0$, which is obtained from the probability $|\langle 0 \mid \Psi\rangle|^{2}=p_{0}$, any $c_{k}$ is determined by means of the expression:

$$
\sqrt{2} c_{0} c_{k}^{*}=\left(p_{1}^{(k)}-p_{2}^{(k)}\right)+i\left(p_{3}^{(k)}-p_{2}^{(k)}\right) .
$$

Thus, the measurement outcomes of these $3(d-1)$ projectors in addition with a previous measurement onto the canonical base $|k\rangle_{k=0}^{d}$, are enough to determine any pure state and certify the a priori assumption of purity. As these projectors do not depend on the nature of the quantum system the tomographic scheme is not restricted to the present setup and it can be in principle implemented for general quantum systems.

\section{CONCLUSION}

Summarizing, we have presented a method that reduces to a minimum the number of measurements for reconstructing all pure quantum states of arbitrary dimension $d$. For this tomographic scheme the outcome probabilities of a total of $4 d-3$ projectors are needed, from which we can also certify if the quantum system is actually in a pure state. Moreover, in the particular case of spatial qudits we propose and experimental implemented a setup that enables to perform this method in a non adaptive way and reducing the number of measurement outcomes to only four, independently of the dimension $d$ of the states to be characterized. We have observed a quite good performance of our implementation at least up to dimension $d=14$, with mean fidelities between the expected and reconstructed states higher than 0.97 in any case.

\section{ACKNOWLEDGMENTS}

We express our gratitude to C. T. Schmiegelow for providing us with the sCMOS camera. This work was supported by UBACyT 20020130100727BA, CONICET PIP $11220150100475 \mathrm{CO}$, and ANPCYT PICT 2014/2432. QPS is in a CONICET Fellowship.
[1] M. Paris and J. Rehacek, Quantum State Estimation, 1st ed. (Springer Publishing Company, Incorporated, 2010).

[2] D. F. V. James, P. G. Kwiat, W. J. Munro, and A. G. White, Phys. Rev. A 64, 052312 (2001)

[3] R. T. Thew, K. Nemoto, A. G. White, and W. J. Munro, Phys. Rev. A 66, 012303 (2002)

[4] W. K. Wootters and B. D. Fields, Annals of Physics 191, 363 (1989)

[5] R. B. A. Adamson and A. M. Steinberg, Phys. Rev. Lett. 105, 030406 (2010)

[6] N. J. Cerf, M. Bourennane, A. Karlsson, and N. Gisin, Phys. Rev. Lett. 88, 127902 (2002).

[7] D. Collins, N. Gisin, N. Linden, S. Massar, and S. Popescu, Phys. Rev. Lett. 88, 040404 (2002).

[8] S. Gröblacher, T. Jennewein, A. Vaziri, G. Weihs, and A. Zeilinger, New J. Phys. 8, 75 (2006)

[9] P. B. Dixon, G. A. Howland, J. Schneeloch, and J. C. Howell, Phys. Rev. Lett. 108, 143603 (2012).

[10] H. J. Lee, S.-K. Choi, and H. S. Park, Scientific Reports 7 (2017), 10.1038/s41598-017-04444-8

11] D. Gross, Y.-K. Liu, S. T. Flammia, S. Becker, and J. Eisert, Phys. Rev. Lett. 105, 150401 (2010).

[12] C. Riofrío, D. Gross, S. Flammia, T. Monz, D. Nigg, R. Blatt, and J. Eisert, Nature Communications 8 (2017), 10.1038/ncomms15305

[13] S. T. Flammia, A. Silberfarb, and C. M. Caves, Foundations of Physics 35, 1985 (2005)

[14] T. Heinosaari, L. Mazzarella, and M. M. Wolf, Communications in Mathematical Physics 318, 355 (2013).
[15] C. Carmeli, T. Heinosaari, J. Schultz, and A. Toigo, EPJ D 69, 179 (2015)

[16] D. Goyeneche, G. Cañas, S. Etcheverry, E. S. Gómez, G. B. Xavier, G. Lima, and A. Delgado, Phys. Rev. Lett. 115, 090401 (2015)

[17] L. Neves, S. Pádua, and C. Saavedra, Phys. Rev. A 69, 042305 (2004)

[18] K. Creath, Progress in Optics 26, 349 (1988).

[19] L. Rebón, C. Iemmi, and S. Ledesma, Optik 124, 5548 (2013).

[20] L. Neves, G. Lima, J. G. Aguirre Gómez, C. H. Monken, C. Saavedra, and S. Pádua, Phys. Rev. Lett. 94, 100501 (2005),

[21] G. Lima, A. Vargas, L. Neves, R. Guzmán, and C. Saavedra, Optics Express 17, 10688 (2009)

[22] V. Krishnaswami, C. J. F. Van Noorden, E. M. M. Manders, and R. A. Hoebe, Opt. Nanoscopy 3, 1 (2014)

[23] J. Leach, M. J. Padgett, S. M. Barnett, S. Franke-Arnold, and J. Courtial, Phys. Rev. Lett. 88, 257901 (2002).

[24] G. Lima, L. Neves, R. Guzmán, E. S. Gómez, W. A. T. Nogueira, A. Delgado, A. Vargas, and C. Saavedra, Optics Express 19, 3542 (2011).

[25] M. Malik, M. Mirhosseini, M. Lavery, J. Leach, M. Padgett, and R. Boyd, Nature Communications 5 (2014), 10.1038/ncomms4115.

[26] M. A. Solís-Prosser, A. Arias, J. J. M. Varga, L. Rebón, S. Ledesma, C. Iemmi, and L. Neves, Opt. Lett. 38, 4762 (2013). 
[27] J. J. M. Varga, L. Rebón, M. A. Solís-Prosser, L. Neves, S. Ledesma, and Iemmi, J. Phys. B 47, 225504 (2014).

[28] J. Leach, R. Warburton, S. Murugkar, M. Edgar, M. Padgett, and R. Boyd, in International Conference on Quantum Information (Optical Society of America, 2011) p. PDPA1.
[29] R. Fickler, M. Krenn, R. Lapkiewicz, S. Ramelow, and A. Zeilinger, Scientific reports 3 (2013).

[30] M. Unternährer, B. Bessire, L. Gasparini, D. Stoppa, and A. Stefanov, Opt. Express 24, 28829 (2016)

[31] R. Jozsa, Journal of Modern Optics 41, 2315 (1994) 